\title{
Kombinatorik, Witz, Rhizom: Prosa als gesteigerte semiotische Interdependenz
}

\section{Erzählung versus Prosa}

Albrecht Koschorke schreibt in seiner 2012 erschienenen Studie zu einer Allgemeinen Erzähltheorie, Wahrheit und Erfindung, eine grundlegende Leistung des Erzählens bestehe darin, semiotische Interdependenzen zu unterbrechen: »Wenn alles mit allem rhizomatisch verbunden scheint, besteht Ordnungsstiftung schon allein in der negativen Operation, semiotische Interdependenz $\mathrm{zu}$ unterbrechen." (Koschorke 2012, 186) Der Erzählung wird damit die Funktion der Komplexitätsreduktion zugesprochen, die es vermag, aus einem unübersichtlichen Geflecht von Bedeutungsverzweigungen und -verknüpfungen einen einzelnen Bedeutungszusammenhang wie ein Schema vor dem Grund hervortreten zu lassen. Narrationen mit ihren Handlungsgefügen und Figurenkonstellationen beruhen auf Zusammenhängen, die über temporale, lokale, figurale und kausallogische Verknüpfungen hergestellt werden. Die Erzähltheorie, allen voran jene nach Genette, hat ein ebenso komplexes wie präzises Kategorien- und Begriffsinventar erarbeitet, mit dessen Hilfe sich diese Verknüpfungen, die einen Erzähltext strukturieren und seine semantische Kohärenz gewährleisten, beschreiben lassen. Wie unterscheidet sich davon Prosa, die sich nicht auf das Moment der Erzählung reduzieren lässt und das Form- und Funktionsparadigma sprengt, nach dem die Gattungspoetik erzählende Literatur ordnet und die Narratologie Formen des Erzählens systematisiert? Eine Prosa, die sich mithin diesen Beschreibungskategorien entzieht? ${ }^{1}$ Besteht Narration, wie Koschorke schreibt, in der ordnungsstiftenden Operation, wuchernde semiotische Zusammenhänge einzudämmen und auf einzelne aktualisierte Bedeutungsrelationen hin stillzustellen, so möchte ich Prosa im Folgenden ausgehend von dieser Definition ex negativo als dasjenige Schreibverfahren begreifen, das semiotische Interdependenzen gerade nicht unterbricht, sondern sich ihrer vielmehr bedient und sie gezielt steigert.

Prosatexte dieser Art zielen damit nicht auf begriffliche Präzision, sondern erodieren im Gegenteil die Begriffe und treiben sie hin zum Unbegrifflichen.

1 Auch der Band Logik der Prosa (Arndt et al. 2012) konzentriert sich neben ästhetischen Verfahren in erster Linie auf die Gattungspoetik und die Erzähltheorie.

Yvonne Al-Taie, Kiel

Ә Open Access. (C) 2021 Yvonne Al-Taie, publiziert von De Gruyter. (cc)BY Dieses Werk ist lizenziert unter einer Creative Commons Namensnennung 4.0 International Lizenz. https://doi.org/10.1515/9783110731569-012 
Hans Blumenberg bestimmt in seiner Theorie der Unbegrifflichkeit die Leistungen des Begriffs unter Rekurs auf Arnold Gehlen als »Entlastung unter den Bedingungen der Reizüberflutung «, wodurch der Begriff eine »Art von Rationalität« ausübe, »die im Begriff der Ökonomie steckt. Jede Leistung ist hier ein Stück Ersparnis« (Blumenberg 2007, 26). Texte der nicht-erzählenden Prosa zeichnen sich hingegen dadurch aus, dass ihre Leistung gerade nicht in ökonomischer Komplexitätsreduktion und entlastender Einschränkung semantischen Potentials besteht, sondern sie im Gegenteil das verwirrende Spiel der semiotischen Interdependenzen auskosten, indem sie die von Diskurs zu Diskurs changierenden Bedeutungen und Bedeutungsrelationen ausstellen, die nicht-semantischen Elemente der Zeichen mittels rhetorischer Figuren hervorheben und dadurch unablässig die Überdeterminiertheit der Begriffe steigern. Sie gestalten eine semiotische Reizüberflutung, die die Leser bisweilen regelrecht zu überfordern scheint.

Dies geschieht in Texten der Prosa, so soll im Folgenden diskutiert werden, auf drei Ebenen: (1.) Durch das Produktivwerden der sprachlichen Ebene selbst. Das sprachliche Material, aus dem die Prosa ihre teilweise ins Extreme gesteigerten extensiven Textkorpora generiert, wird in seinen vielstelligen semantischen und klanglichen Bezügen erfasst und aktiviert. Von diesem Potential der sprachlichen Zeichen ausgehend etablieren Texte der Prosa (2.) Verweisketten, die auch die Inhaltsebene betreffen und Gegenstände, Themen und Diskurse durch ein den gesamten Textverlauf überspannendes Beziehungsgeflecht miteinander verknüpfen und in produktive Beziehung zueinander treten lassen. Schließlich entfalten Texte der Prosa (3.) als Metaebene dieses sprachlichen Beziehungsgeflechts selbstreferentielle Textstrukturen.

Ralf Simon hat für dieses Schreibverfahren den Begriff der »Durcheinanderprosa " (Simon 2014) geprägt. Ausgehend von Foucaults in Les mots et les choses grundgelegter Unterscheidung zwischen drei historischen Etappen der Wissensgeschichte - den auf Ähnlichkeitsbeziehungen beruhenden Epistemen der Renaissance, den auf einem zweistelligen, repräsentationalen Zeichenmodell aufbauenden Epistemen der Aufklärung und den am Paradigma der Transzendentalität orientierten Epistemen des Idealismus - entwickelt Simon die These, dass künstlerische Darstellungsverfahren das Potential bergen, alle drei dieser bei Foucault auf einer historisch-chronologischen Achse angeordneten Modelle des epistemologischen Weltbezugs in einem einzigen Werk miteinander zu verschränken (Simon 2014, 20). Dabei, so Simons These weiter, affizieren die Episteme der Ähnlichkeit die anderen Formen der Zeichenrelation. Foucaults Bildanalyse von Velázquez Las Meninas dekonstruktivistisch gegen den Strich lesend, konstatiert Simon für die Kunst die Möglichkeit, gegenüber der Geschichte der Wissensformen eine »Gleichzeitigkeit von epistemischen Systemzusammenhängen« (ebd.) zu etablieren, ein »Zugleich der Epistemai, ihr eigentlich systemwidriges Interagie- 
ren« (ebd., 21), das auf einem re-entry der Episteme der Ähnlichkeit in die beiden anderen Episteme - der Repräsentation und der Transzendentalität - beruht, um scheinbar logikwidrige Gleichzeitigkeit zu bewerkstelligen.

Meine Überlegungen folgen einem ähnlichen Ansatz, wobei ich von drei historischen Konzepten der (literarischen) Zeichenverwendung ausgehen möchte, die jeweils die semiotische Interdependenzsteigerung zum Ziel haben, dabei aber ähnlich, wie Simon dies für die Foucault'schen Episteme aufzeigt - auf unterschiedlichen Ebenen wirksam werden. Im Folgenden werde ich die Kombinatorik als ein Zeichenmodell der Frühen Neuzeit, den Witz und die Ironie als das transzendentalphilosophische Weiterdenken der Kombinatorik in der Romantik und schließlich das Rhizom als eine Figur der Zeichen- und Diskusverschränkung in der Postmoderne unterscheiden. Während die frühneuzeitliche Kombinatorik auf der Ebene der Materialität der Zeichen operiert, fügen frühromantischer Witz und Ironie die selbstreferentielle Ebene hinzu und Deleuzes und Guattaris Konzept des Rhizoms bringt schließlich die Wissensbestände - oder die referentielle Ebene - mit ins Spiel. Alle drei Ebenen der semiotischen Interdependenzsteigerung - die kombinatorische Ebene, die selbstreferentielle Ebene und die referentielle Ebene -, so die These, die davon ausgehend entfaltet und anhand eines Beispiels diskutiert werden soll, wirken in Texten der Prosa zusammen.

Was bei Foucault als drei unterschiedliche Wissensformen erscheint, möchte ich so vielmehr in Anlehnung an Simons Konzept der »Durcheinanderprosa» als drei Ebenen der Zeichenverwendung begreifen, die sich in der Prosa überlagern und so die semiotischen Interdependenzen steigern. Sie werden damit nicht als epistemologische Alternativen gedacht, sondern als ein Zugleich sichtbar gemacht, in dem die Form oder Materialität der Signifikanten, die durch sie repräsentierten Inhalte und schließlich das transzendental-selbstreflexive Moment koexistieren. Das kombinatorische Moment der Zeichenrelation bildet dabei die Grundlage der beiden nachfolgenden Konzepte des Witzes beziehungsweise der Ironie als Form transzendentaler Selbstbezüglichkeit und des Rhizoms als Organisationsform unterschiedlicher Wissensdiskurse, die sich der kombinatorischen Zeichenverwendung jeweils bedienen. Die Kombinatorik verstehe ich dabei nicht als an eine bestimmte Wissensform gebunden, wie etwa an die Episteme der Ähnlichkeit, sondern als eine Art des Zeichengebrauchs, an die sich unterschiedliche Wissensformationen anlagern können.

Ich möchte zunächst in einigen wenigen historischen Etappen diese drei Verfahren kurz skizzieren, um daran anschließend das Zusammenspiel kombinatorisch-zeichenrelationaler, transzendental-selbstreferentieller und rhizomatisch-diskursverschränkender Verfahren in der Prosa am Beispiel von Ulrike Draesners Mein Hiddensee nachzuvollziehen. 


\section{Kombinatorik - Steigerung der Zeichenrelationen}

Das erste Foucault'sche Wissensmodell ist jenes der Ähnlichkeit. Es ist zunächst ein epistemologisches Konzept und zielt auf die Verhältnisse realer Gegenstände; gleichwohl erschließen sich diese Verhältnisse erst, wenn sie für den Menschen als Zeichen lesbar werden: »Die Welt der Ähnlichkeit kann nur eine bezeichnete Welt sein.« (Foucault 1974, 57) Foucault rekurriert hier auf die Signaturenlehren der Frühen Neuzeit, in deren Denken Zeichenrelationen und Relationen des Bezeichneten in analoger Beziehung zueinander stehen, wie es sich in den magischhermeneutischen Lehren, etwa der Kabbala, spiegelt (ebd., 57-74). Nach Foucault findet das Denken der Ähnlichkeit im Barock sein Ende und wird im siebzehnten Jahrhundert von einer Episteme der Repräsentation abgelöst, die er auch im kombinatorischen Zeichenverständnis, etwa bei Leibniz, realisiert sieht. Die Kombinatorik muss hingegen weiter gefasst und von den Epistemen der Aufklärung losgelöst betrachtet werden. Bei ihr handelt es sich um ein Verfahren seriell-systematischer Zeichenpermutation, das sich bereits in der Antike und im Spätmittelalter findet, in der Frühen Neuzeit besonders große Wirksamkeit entfaltet hat ${ }^{2}$ und für die Schriftauslegung der Kabbala von großer Bedeutung ist, wo sie zur hermeneutischen Interpretation auch der nicht-semantischen Elemente der Sprache herangezogen wird. ${ }^{3}$ Im Barock entstehen kombinatorische Sprachund Dichtungstheorien, unter anderem in Justus Georg Schottelius' Ausführlicher Arbeit von der teutschen HaubtSprache von 1663, bei Philipp von Zesen und Georg Harsdörffer (Neubauer 1978, 28). Gerade in ihrer literarischen Adaption hat die Kombinatorik weit über die Frühe Neuzeit hinaus Wirksamkeit entfaltet. Sie soll hier als die erste grundlegende Ebene semiotischer Interdependenzsteigerung verstanden werden, die für die Schreibweisen der Prosa relevant ist. Dabei verstehe ich die Kombinatorik - anders als Foucault - nicht als an eine bestimmte Wissensform gebunden, sondern als ein Verfahren, das auf der Ebene

2 Kombinatorische Lehren verbreiteten sich seit Raimund Lulls Ars magna generaliset et ultima (1305-1308) und der gekürzten Ars brevis von 1308 durch Lulls Schüler, die seine Werke ab dem fünfzehnten Jahrhundert im Druck herausgaben und an den Universitäten lehrten, so etwa Nikolaus von Kues und Agrippa von Nettesheim. Im siebzehnten Jahrhundert werden Athanasius Kircher mit der Ars Magna Sciendi (1669) und Gottfried Wilhelm Leibniz mit De Arte Combinatoria (1666) wichtige Vertreter kombinatorischer Lehren. Ferner werden Lehren der Kabbala mit der Kombinatorik in Verbindung gebracht, exemplarisch dafür steht Pico della Mirandolas Apologia als ars combinandi. (Neubauer 1978, 19-21; Breitbach 2005, 330) 3 »One of the most striking characteristics of the Hebrew kabbalah, and one that had an impact on the Christian kabbalah, is the extension of meaning to non-semantic aspects of language.«(Dan 1997, 70) 
der Signifikantenverknüpfung produktiv wird. ${ }^{4}$ Die Kombinatorik ist ein Verfahren, das Ordnungsmuster semiotischer Konstellationen jenseits ihrer semantisch-syntaktischen Verknüpfung erprobt und systematisiert. Ihren Ausgangspunkt nimmt die Kombinatorik bei den Wissensformen der Analogie und der Ähnlichkeit. ${ }^{5}$ Sie ist dabei jedoch nicht notwendig an die Episteme der Ähnlichkeit gekoppelt und kann mithin als re-entry auch in anderen Wissensformationen - etwa in der transzendentalen oder in der repräsentationalen - wirksam werden und zu einer Komplexitätssteigerung führen, wie sie Texte der Prosa auszeichnet. Kombinatorik soll im Folgenden als Grundform vorwiegend nicht-erzählender Schreibweisen der Prosa betrachtet werden.

Als ein Verfahren der rein wortbezogenen frühneuzeitlichen Grammatik basierte die Kombinatorik vor allem auf der Arbeit mit Stammwörtern und hat ihren Niederschlag in lexikographischen Projekten der Stammwörterbücher gefunden. Als Grundbaustein des Stammworts gilt wiederum der Stammbuchstabe; ${ }^{6}$ phraseologische Überlegungen kommen quasi nicht vor (Hundt 2000, 254). Die grammatikalische Grundannahme, »dass die deutsche Sprache über eine geschlossene Klasse von Stammwörtern verfüge, aus denen sich alle nur denkbaren Kombinationen durch die Wortbildung herstellen lassen« (ebd., 65), bildet den Ausgangs-

4 Anders als dies Foucaults Darstellung nahelegt, ist die Kombinatorik nicht notwendig an ein bestimmtes Wissensmodell gebunden. Während Foucault die Kombinatorik unter Rekurs auf deren Anwendung etwa bei Leibniz und der Logik von Port-Royal in den Epistemen der Repräsentation des siebzehnten Jahrhunderts und der Aufklärung verortet, zeigt Hundt (2000, 104, 216) auf, wie eng die Kombinatorik mit den Epistemen der Ähnlichkeit verknüpft sein kann, etwa in ihren kabbalistischen Anwendungen. Markus Hundt weist ferner darauf hin, dass es für die Kombinatorik in den barocken Sprachlehren nicht mehr als abgesichert gelten kann, dass sie noch an ein magisches Ähnlichkeitsdenken gekoppelt ist, oder ob darin nicht vielmehr spielerisch darauf Bezug genommen wird, während sich bereits ein binäres Zeichenmodell der Repräsentation durchgesetzt hat.

5 Die »welt- und erkenntniskonstitutive Funktion der Sprache« (Hundt 2000, 216, 271, u.ö.) im frühneuzeitlichen Denken darf nicht übersehen werden. Man ging davon aus, dass Stammwörter in einer nicht-arbiträren Verbindung zu den von ihnen bezeichneten Gegenständen stehen, was wiederum - ausgehend von der Vorstellung, dass Laut und Buchstabe eine Einheit ausmachen - aus der lautmalerischen und lautsymbolischen Bedeutung der einzelnen Buchstaben abgeleitet wurde. Laut und Buchstabe wurden noch im siebzehnten Jahrhundert als Einheit gedacht, so dass Hundt $(2000,183)$ auch von »Graphonemen « spricht. Entsprechend versuchte man auch den Fachwortschatz onomatopoetisch zu erklären. Zentral für die Episteme der Ähnlichkeit ist ferner die Grundvorstellung, dass sich in den aufgedeckten Ähnlichkeitsrelationen der Wörter die Beziehung der durch sie bezeichneten realweltlichen Gegenstände abbildet. »Durch die Gemeinsamkeit des Buchstabenmaterials war auch eine prinzipielle Verbundenheit der Inhalte gegeben.« (ebd., 218)

6 So bei Schottelius (Hundt 2000, 187-193). 
punkt permutativ-kombinatorischer Verfahren, die sich ebenso in lexikographischgrammatischer Ausrichtung wie in ludistischer Ausprägung finden.

Die permutativen Verfahren (Anagrammatik, Palindrome, Proteusverse, Leselabyrinthe, carmen infinitum $)^{7}$ können sich sowohl auf den Lese- als auch auf den Schreibprozess beziehen und führen $\mathrm{zu}$ einer seriellen Textproduktion, für die schon im Mittelalter und in der Frühen Neuzeit bisweilen technische Apparaturen zur Anwendung kamen. ${ }^{8}$

Sprachkombinatorische Verfahren operieren auf der Ebene der Signifikanten. Die Operationen zielen vor allem auf die analytische Zergliederung der Begriffe und die anagrammatische Reorganisation der Zeichen. Ihre Methoden der Textgenerierung und Permutation von sich scheinbar bis ins Unendliche überlagernden Bedeutungsschichten rekurrieren ausschließlich aufs semiotische Material, das in Graphemen, Phonemen und lexikalischen Einheiten die Grundelemente der Textproduktion bereitstellt und durch immer neue Arrangements unaufhörlich neue Sinnschichten an das expandierende Textgebilde anlagert. Die spielerischen Formen, wie sie etwa in Harsdörffers Frauenzimmergesprächsspielen geschildert werden, bestehen in sprachkombinatorischen Verfahren, etwa in der Suche nach möglichst vielen Ableitungen, Derivationsformen, Komposita, Negativformen oder Sprichwörtern von vorgegebenen Stammwörtern (Hundt 2000, 272-275). Das Ziel aller dieser Formen sprachspielerischer Geselligkeitskultur bestand darin, das »Wortbildungspotential eines Stammwortes aufzudecken« (ebd., 274).

Die Kombinatorik ist dabei strikt systematisch organisiert und präsentiert sich in Form von regelbasierten Tafeln und Diagrammen, die alle möglichen Zuordnungen und Zusammenstellungen eines begrenzten Sets an Grundbegriffen abzubilden versuchen. Verfahren kombinatorischen Sprachgebrauchs sind mit enzyklopädischen Verfahren verwandt, die auf Vollständigkeit des erfassten Sprachmaterials abzielen und dieses in der Regel nach sprachimmanenten Kriterien sortieren. Olaf Breitbach spricht in diesem Zusammenhang von einem »Instrumentarium einer schlüssigen Totalrepräsentation der uns möglichen Begriffsbezüge« (Breitbach 2005, 329).

Allen Spielarten dieses Verfahrens liegt ein Grundprinzip der Textproduktion zugrunde, das nicht vom inhaltlich-logischen Aufbau eines Textes über Aussagen und Aussageverknüpfungen ausgeht, sondern Texte von ihren (kleinsten) sprachlichen Elementen her denkt und deren Potential zu Arrangements immer neuer Textkonstellationen auslotet. Texte werden dergestalt zu Konstellationen

$7 \mathrm{Zu}$ diesen Verfahren und ihren Spielarten ausführlich Ernst 1992.

8 Solche Apparaturen gab es etwa in Form von Drehzylindern oder als Textscheiben mit gegeneinander verschiebbaren konzentrischen Ringen, die eine mathematisch exakt berechenbare Anzahl von Begriffskombinationen ermöglichten (Ernst 1992, 243f.). 
sprachlichen Materials, die die Zusammenschau erschöpfender Bedeutungsvarianten auf Basis eines begrenzten Sets an sprachlichen Grundbausteinen auskosten.

Der Komplexität dieser Verfahren und der überbordenden Fülle an dieserart generierbaren Bedeutungsvariationen mag es geschuldet sein, dass sie in erster Linie in der Lyrik zur Anwendung kamen - im Barock ${ }^{9}$ ebenso wie in der avantgardistischen und experimentellen Dichtung des zwanzigsten Jahrhunderts (Ernst 1992, 225-269). Insbesondere auf exhaustive Vollständigkeit abzielende Umsetzungen solcher Schreibformen fordern beinahe notwendig eine das sprachliche Material beschränkende Kleinform, wie sie die Lyrik darstellt. Mit dem nahezu unkontrollierbar werdenden Anschwellen immer neuer Bedeutungskonstellationen wohnt der Kombinatorik zugleich aber auch ein Moment inne, das sie zu einem präferierten Produktionsverfahren extensiver Prosatexte werden lässt. ${ }^{10}$

Dabei operieren kombinatorische Verfahren auf der Ebene der Zeichen, sie stellen das selbstreferentielle Spiel der Zeichenverkettungen aus und lassen den Textkörper in immer neuen rekursiven Schleifen semiotischer Variation anschwellen. Diese gesteigerte Aufmerksamkeit für das sprachliche Zeichen als Baustein des Textes und für die Möglichkeiten der unaufhörlichen Bedeutungsgenerierung durch das Umstellen und Rekombinieren dieser Elemente ist grundlegend zum Verständnis der hier in Rede stehenden Prosa. Prosa zielt damit anders als Erzählungen nicht in erster Linie auf Handlungszusammenhänge, sondern auf Zeichenzusammenhänge.

Von diesen Befunden ausgehend soll nachfolgend aufgezeigt werden, dass die Kombinatorik als ein auf Signifikantenebene wirksames Modell der Organisation und Interaktion von Zeichen verstanden werden kann, dessen sich Texte der Prosa bedienen, um semiotische Interdependenzen zu steigern. Dabei lassen sich kombinatorische Verfahren des Zeichengebrauchs als re-entry in andere Wissensformationen als der ähnlichkeitsbasierten integrieren, so dass diese Texte mit mehreren Wissensordnungen zugleich operieren. Mit dem romantischen Witz

\footnotetext{
9 Poetische Anwendung fand die Kombinatorik unter anderem in den sogenannten Proteusversen, in denen die Wörter seriell ihre Position im Satz tauschen, so dass eine Text- oder Gedichtserie mit immer neuen Bedeutungsverschiebungen entsteht. Der Name Proteusverse geht auf die mythologische Überlieferung zurück, wonach sich der antike Proteus in verschiedene Elemente und Lebewesen verwandelt habe. Prototypisch für die Gattung sind die Proteusverse des Thomas Lansius, die auch bei Leibniz zitiert werden. Der erste Vers lautet: LEX, REX, GREX, RES, SPES, JUS, THUS, SAL, SOL, LUX, LAUS. Lansius berechnet über 39 Millionen möglicher Permutationen für diese Verse. (Lansius 1620; Ernst 1992, 240). Bei Harsdörffer und Quirinus Kuhlmann finden sich Variationen dieses kombinatorischen Versmodells (Neubauer 1978, 27).

10 Ernst 1992, 249-252 verweist exemplarisch auf Umberto Ecos Der Name der Rose. Hier ließen sich zahlreiche weitere Autoren anführen, etwa James Joyce oder Arno Schmidt.
} 
und der romantischen Ironie sowie der postmodernen Figur des Rhizoms sollen zwei solcher Darstellungsformen aufgezeigt werden, die jeweils eine andere Episteme auf Grundlage der Kombinatorik entfalten.

\section{Witz: Transzendentale Steigerung der Kombinatorik}

Die Anwendung kombinatorischer Verfahren wird in Texten der Prosa mit einer zweiten, als transzendental oder metareflexiv zu bezeichnenden Ebene verknüpft, wie Foucault sie für die Episteme des Idealismus beschreibt und wie Simon sie als ein Merkmal der »Durcheinanderprosa« kennzeichnet. Mit dem Witz und der Ironie als den beiden zentralen Denkfiguren der romantischen Poetik sollen im Folgenden zwei Schreibverfahren in den Fokus gerückt werden, die die kombinatorische Zeichenverwendung dazu heranziehen, um den Texten ein selbstreflexives Moment einzuschreiben.

Die Frühromantik greift das Textmodell der ars combinatoria auf, verbindet es jedoch in der Figur des Witzes mit einem selbstreflexiven Moment, sodass der textuellen Bedeutungsgenerierung und -steigerung über Ähnlichkeitsrelationen eine metareflexive Dimension hinzugefügt wird. Friedrich Schlegel ${ }^{11}$ stellt die Verbindung zwischen Witz und ars combinatoria in seinen Notizen an verschiedenen Stellen explizit her, am deutlichsten vielleicht in der Formulierung: »Der höchste Witz wäre die wahre ling.[ua] char.[akteristica] universalis und ZUGLEICH die ars combinat[oria]« (KFSA XVIII, 281) oder in Ausdehnung dieser Reihe der Äquivalenzen auf die Kritik und die Erfindungskunst: »Witz, ars combinat.[oria], Kritik, Erfindungskunst, ist alles einerlei.« (KFSA XVIII, 124) So verschwistern sich im kombinatorischen Witz zugleich zwei Schreibweisen, um deren Verbindung es dem frühromantischen Denken schlechthin zu tun ist: Kritik und poetisch-dichterische Erfindung. Dieses Doppel von Poetizität und kritischer (Selbst-)Reflexion ist auch im Konzept der romantischen Ironie eingefangen. Ironie legt ein Zugleich zweier Bedeutungsregister der Zeichen frei, in dem die Zeichen sowohl die Bedeutung ihrer im Text aktualisierten Semantiken entfalten als auch in einer »ironische[n] Überdeterminierung " polyseme Bedeutungsspielräume aktivieren (Frank 1989, 366). Witz und Ironie sind zwei bei Friedrich Schlegel eng aufeinander bezogene Konzepte, die er in einer Notiz wie folgt unterscheidet: »Ironie ist inner-

11 Zur Nähe der frühromantischen Poetik zu einer Theorie der Prosa und zu Friedrich Schlegels Nachdenken über eine solche: Simon 2013, 202-209. 
lich; der Witz nur die Erscheinung derselben.« (KFSA XVIII, 203) Die hier versuchte Differenzierung ließe sich dahingehend reformulieren, dass Ironie in erster Linie das transzendentalpoetische Prinzip einer selbstreflexiven Poesie der Poesie meint, während Witz auf die Form ihrer sprachlichen Darstellung bezogen ist. ${ }^{12}$

Witz und Ironie werden dabei nicht ausschließlich und auch nicht in erster Linie rhetorisch gedacht, sondern unter Bezugnahme auf Kants Transzendentalphilosophie als grundlegende Verfahren eines poetischen Schreibens entworfen, das sich seiner selbst bewusst wird. ${ }^{13}$ >Transzendentalpoesie und Transzendentalphilosophie konvergieren im Begriff der `Kritikı als deren zentrales Charakteristikum, wonach die Darstellung die Bedingungen des Darstellens immer schon mitreflektiert. Die Grundgedanken dieses romantischen Textverständnisses sind im berühmten 238. Athenäums-Fragment niedergelegt, dessen Wortlaut hier noch einmal in Erinnerung gerufen sei:

Es gibt eine Poesie, deren eins und alles das Verhältnis des Idealen und des Realen ist, und also nach der Analogie der philosophischen Kunstsprache Transzendentalpoesie heißen müßte. Sie beginnt als Satire mit der absoluten Verschiedenheit des Idealen und Realen, schwebt als Elegie in der Mitte, und endigt als Idylle mit der absoluten Identität beider. So wie man aber wenig auf eine Transzendentalphilosophie legen würde, die nicht kritisch wäre, nicht auch das Produzierende mit dem Produkt darstellte, und im System der transzendentalen Gedanken zugleich eine Charakteristik des transzendentalen Denkens enthielte: so sollte wohl auch jene Poesie die in modernen Dichtern nicht selten transzendentalen Materialien und Vorübungen zu einer poetischen Theorie des Dichtungsvermögens mit der künstlerischen Reflexion und schönen Selbstbespiegelung, die sich im Pindar, den lyrischen Fragmenten der Griechen, und der alten Elegie, unter den Neuern aber in Goethe findet, vereinigen und in jeder ihrer Darstellungen sich selbst mit darstellen, und überall zugleich Poesie und Poesie der Poesie sein.

(KFSA II, 204)

Die Schreibverfahren, in denen sich diese Verbindung aus Philosophie und Dichtung vollzieht, sind der Witz und die Ironie. Schon in einem Lyceums-Fragment begreift Friedrich Schlegel die Ironie als eine philosophische Darstellungsform: »Die Philosophie ist die eigentliche Heimat der Ironie« (KFSA II, 152) und präzisiert seine Aussage hinsichtlich einer philosophischen Darstellungsform, die nicht in einem System gründet und dem Literarischen damit besonders nahesteht: »[D]enn überall wo in mündlichen oder geschriebenen Gesprächen, und nur nicht ganz systematisch philosophiert wird, soll man Ironie leisten und fordern«. (KFSA II, 152) Ähnlich notiert er über den Witz: »Aller W[it]z hat Verwandtschaft mit $\varphi \sigma[$ Philosophie].« (KFSA XVIII, 27)

12 Zum Verhältnis von Witz und Ironie bei Friedrich Schlegel: Zovko 2017.

13 Wie Manfred Frank $(1989,296)$ schreibt: "Allegorie und Witz sind die Blick- und WendePunkte der Reflexion«. 
Vielleicht ist Friedrich Schlegels Idee des Witzes nirgendwo sonst so geistreich wie elegant zum Ausdruck gebracht wie im 220. Athenäums-Fragment:

Ist aller Witz Prinzip und Organ der Universalphilosophie, und alle Philosophie nichts anderes als der Geist der Universalität, die Wissenschaft aller sich ewig mischenden und wieder trennenden Wissenschaften, eine logische Chemie: so ist der Wert und die Würde jenes absoluten, enthusiastischen, durch und durch materialen Witzes, worin Baco und Leibniz, die Häupter der scholastischen Prosa, jener einer der ersten, dieser einer der größten Virtuosen war, unendlich. Die wichtigsten wissenschaftlichen Entdeckungen sind bonmots der Gattung. Das sind sie durch die überraschende Zufälligkeit ihrer Entstehung, durch das Kombinatorische des Gedankens, und durch das Barocke des hingeworfenen Ausdrucks. Doch sind sie dem Gehalt nach freilich weit mehr als die sich in Nichts auflösende Erwartung des rein poetischen Witzes. Die besten sind echappées de vue ins Unendliche.

(KFSA II, 200)

Die gesamten Aussagen über den Witz gruppieren sich um die Nennung zweier beispielhafter Vertreter der Gattung - Bacon und Leibniz - als den »Häupter[n] der scholastischen Prosa«. Der Witz der philosophischen Prosa gilt dabei dem poetischen Witz aufgrund seines epistemischen Gehalts als überlegen. Witz soll ein erkenntniskritisches Potential enthalten, das auf der nicht logisch organisierten Zusammenführung heterogener Gegenstände und Wissensbereiche beruht und deren sprachliche Darstellung den aus dem Barock bekannten kombinatorischen Formen entspricht. Der philosophische Witz der Prosa gründet dergestalt in Momenten des beständigen Formwechsels. Philosophische Reflexionen schreiben sich Texten der Prosa mithin anders als in Erzählungen nicht in erster Linie über den Handlungsverlauf unterbrechende - digressive Figuren- oder Erzählerreden in den Text ein, sondern bilden das metareflexive Moment einer kombinatorischen Sprachverwendung.

Was sich in der philosophischen Verwendung des Witzes bereits andeutet dass er im kombinatorischen Spiel Inhalte verknüpft und damit auch welthaltig ist - entfalten Deleuze und Guattari unter Rekurs auf die Denkfigur des Rhizoms als ein postmodernes Textverständnis.

\section{Rhizom: Diskursive Steigerung der Kombinatorik}

Es hat sich gezeigt, dass die Kombinatorik zwar die Steigerung semiotischer Interdependenzen denkt und in ihren Verfahren operativ ausgestaltet, dabei jedoch von klaren, zum Teil hierarchisch strukturierten Ordnungsmustern ausgeht. Dieses Denkmodell artikuliert sich in der wirkmächtigen Wissensfigur des arbor scientiae beziehungsweise des arbor porphyriana, deren Baumdiagramm für nachfolgende Adaptionen kombinatorischer Lehren und epistemologischer Ordnungsmuster 
häufig wegweisend blieb. Den Strukturbildern des Baumes oder der Wurzel mit ihren binären Ordnungsprinzipien möchten Gilles Deleuze und Félix Guattari ein ebenso der Natur entlehntes, alternatives Denkmodell entgegensetzen, das sie im Rhizom als einer biologischen Struktur finden, die ins Form- oder Strukturlose drängt: »Das Rhizom selbst kann die verschiedensten Formen annehmen, von der Verästelung und Ausbreitung nach allen Richtungen an der Oberfläche bis zur Verdichtung in Knollen und Knötchen.« (Deleuze und Guattari 1977, 11)

Bereits Koschorkes Formulierung: »Wenn alles mit allem rhizomatisch verbunden scheint«, enthält eine Anspielung auf Deleuzes und Guattaris Essay Rhizom, der den Text jenseits hierarchisch-logischer Ordnungsmuster zu denken versucht. Der zweite Teil von Koschorkes Satz: »besteht Ordnungsstiftung schon allein in der negativen Operation, semiotische Interdependenz zu unterbrechen « (Koschorke 2012, 186), gilt für den rhizomatischen Text freilich nicht mehr. Der Text als Rhizom gedacht folgt nicht den kohärenten Handlungsketten der Erzählung. Mehr noch: Die strukturale Figur des Rhizoms erlaubt es, das kombinatorische Moment aus dem Ordnungsdenken der frühneuzeitlichen Kombinatorik herauszulösen und die kombinatorische Dynamik stattdessen in semiotischen Systemen zu erfassen, die nicht-hierarchischen Mustern folgen und damit einer weiteren Komplexitätssteigerung dienen. Es bildet eine Steigerung von Interdependenzen bei gleichzeitigem Verzicht auf lineare Ordnungsmuster ab.

Auf Basis der Kernprinzipien Konnexion und Heterogenität leistet das Rhizom eine unaufhörliche Verknüpfung heterogenen Materials, die zur semiotischen Interdependenzsteigerung drängt. In den Worten Deleuzes und Guattaris:

Ein Rhizom verknüpft unaufhörlich semiotische Kettenteile, Machtorganisationen, Ereignisse in Kunst, Wissenschaft und gesellschaftlichen Kämpfen. Ein semiotisches Kettenglied gleicht einem Tuberkel, einer Agglomeration von mimischen und gestischen, Sprech-, Wahrnehmungs- und Denkakten: es gibt keine Sprache an sich, keine Universalität der Sprache, sondern einen Wettstreit von Dialekten, Mundarten, Jargons und Fachsprachen.

(Deleuze und Guattari 1977, 12)

Oder, wie Hans-Thies Lehmann es ausdrückt, es vollzieht sich in der Rhizomatik »die Zerstörung des klischierten, wenn auch von der Wissenschaft vielleicht beglaubigten Rasters der Weltbeschreibung zugunsten einer Neues produzierenden Wahrnehmung, in der das Heterogene sich verbinden kann.« (Lehmann 1984, 544) Die Zeichen verdichten sich im Rhizom und pluralisieren sich zu Zeichensystemen, die ihren jeweiligen Eigenwert behaupten, ohne feste Ordnungspunkte innerhalb einer Struktur zu bilden: »In einem Rhizom gibt es keine Punkte oder Positionen wie etwa in einer Struktur, einem Baum oder einer Wurzel. Es gibt nichts als Linien.« (Deleuze und Guattari 1977, 14) Aus diesem fehlenden Zentrum leiten sich wiederum die Segmentierungslinien her, die es erlauben, das Rhizom 
»an jeder beliebigen Stelle« (ebd., 16) aufzubrechen, ohne es zu vernichten; vielmehr wuchert es entlang dieser Linien weiter. Für Texte der Prosa bedeutet dies, dass sie anders als narrative Texte weniger an Handlungslogiken und -folgen gebunden sind. Sie weisen gegenüber Erzähltexten eine weit größere Toleranz gegenüber Umstellung, Streichung und Ergänzung von Textelementen und -segmenten auf.

Während die Kombinatorik auf der Ebene der Zeichenrelationen operiert und der romantische Witz dieser das Moment der Selbstreferentialität hinzufügt, integrieren Deleuze und Guattari mit ihrem Begriff des Rhizoms die signifikative Ebene ins Spiel der Zeichen. Mit dem Rhizom führen Deleuze und Guattari eine Denkfigur der komplexen, nicht-linearen und nicht-kausallogisch organisierten Zeichenverschaltung ein, die als eine Organisationsform von Wissen Gültigkeit gewinnt. ${ }^{14}$ Dieses Ausgreifen der semiotischen Form auf die Inhalte markieren sie deutlich in Abgrenzung zu Chomskys linguistischem Baum:

In einem Rhizom dagegen verweist nicht jeder Strang notwendig auf einen linguistischen Strang: semiotische Kettenglieder aller Art sind dort nach den verschiedensten Codierungsarten mit politischen, ökonomischen und biologischen Kettengliedern verknüpft; es werden also nicht nur ganz unterschiedliche Zeichensysteme ins Spiel gebracht, sondern auch verschiedene Arten von Sachverhalten.

(Deleuze und Guattari 1977, 12)

Der Unterschied zum Repräsentationsmodell der Aufklärung besteht darin, dass Deleuze und Guattari in ihrem poststrukturalistischen Textmodell das re-entry einer kombinatorischen Zeichenverwendung in ihr Modell der Wissensrepräsentation bereits vollzogen haben. So denken sie die Kombinatorik nicht rein auf der Ebene eines selbstreferentiellen Spiels der Signifikanten, sondern sehen diese in Abhängigkeit zum Wirklichkeitsbezug des Textes: »Die Kombinationen, Permutationen und Gebrauchsweisen sind dem Buch nie inhärent, sondern hängen von seinen Verbindungen mit diesem oder jenem Außen ab.« (Deleuze und Guattari 1977, 40) Die Unterscheidung zwischen Wirklichkeit, deren semiotischer Repräsentation und selbstreferentieller Reflexion eines Autorsubjekts erklären Deleuze und Guattari im rhizomatischen Text für aufgehoben. Texte als Rhizome, so könnte man auch sagen, sind dergestalt »Durcheinanderprosa

In ihrer Vielheit bearbeitet eine Verkettung zwangsläufig zugleich semiotische, materielle und gesellschaftliche Ströme [...]. Es gibt keine Dreiteilung mehr zwischen einem Feld der Realität: der Welt, einem Feld der Repräsentation: dem Buch und einem Feld der Subjektivität: dem Autor.

(Deleuze und Guattari 1977, 36)

14 So vermerken sie in ihren historischen Perspektivierungen: „Nietzsches Aphorismen brechen die lineare Einheit des Wissens auf« (Deleuze und Guattari 1977, 10). 
Damit kann die Figur des Rhizoms auch als Selbstbeschreibungsmodell einer Episteme der Moderne betrachtet werden, wie Simon (2014) sie rekonstruiert. Dieses Ineinandergreifen aller drei Zeichenfunktionen führt im Prosatext $\mathrm{zu}$ einer Verdichtung, in der das Wortmaterial sein semiotisches Potential auf allen drei Ebenen zugleich entfaltet: Das Wortmaterial wird zugleich für das kombinatorische Spiel mit Ähnlichkeiten genutzt, durch die referentielle Funktion wird Wirklichkeit in narrativer, beschreibender oder argumentierender Weise dargestellt und in transzendentaler Funktion wird die Gebundenheit der Darstellung an den Zeichengebrauch reflektiert. Dies soll im Folgenden in einer exemplarischen Lektüre von Ulrike Draesners Mein Hiddensee nachvollzogen werden.

\section{Ulrike Draesners kombinatorische Prosa: Mein Hiddensee}

In Ulrike Draesners 2015 erschienenem Prosaband Mein Hiddensee überlagern sich im Streifzug einer Mutter mit ihrem Kind über die Insel Erinnerungen an eine gescheiterte Liebe, ${ }^{15}$ Familiengeschichte, Rekonstruktion historischer Epochen und Ereignisse, die sich der Insellandschaft eingeschrieben haben, mit Naturbeobachtungen und fachterminologischer Naturbenennung; dabei behauptet die Sprache unaufhörlich ihre Eigenlogik, die der referentiellen Darstellung die nicht-semantischen, akustischen und kombinatorischen Verbindungen ihrer Signifikanten aufprägt. Narrative Passagen und stärker wort- und bildbezogenes nature writing wechseln sich ab. ${ }^{16}$ Immer wieder mündet der Text dabei in Reflexionen der Möglichkeiten, Grenzen und Eigengesetzlichkeiten der Sprache, mit der die Wissensund Wahrnehmungsbestände kartiert werden. Die meist nur wenige Seiten langen Kapitel tragen teils referentielle Überschriften, die auf topographische Orte oder Jahreszahlen verweisen, teils auf menschliche Beziehungssituationen hindeuten und teils das visuell-bildliche Wahrnehmen und Darstellen in den Fokus rücken,

$15 \mathrm{Zu}$ diesem wiederkehrenden Thema in Draesners Werken: Leeder 2008a und 2008b.

16 Es sind die nicht-erzählenden Passagen des Buches, die es zu einem hier in Rede stehenden Text der Prosa machen und an denen eine am Paradigma des Erzählens orientierte Literaturkritik Anstoß nimmt (Roßbach 2015). Mit dem Konzept und dem Schreibverfahren des nature writing beschäftigt sich Draesner auch in einer ihrer Frankfurter Poetikvorlesungen unter dem Titel Vom zärtlichen Ernst der Welt. Nature Writing (Draesner 2018). Dabei reflektiert sie das Konzept des nature writing auch im Text selbst: »Nature writing. Natur schreiben? Schreiben nach >der Natur«? Wahrnehmen. Sprechen. Durch das Sprechen wahrnehmen. Sich mithilfe des Sprechens zwingen, die Augen aufzureißen. Das Gehirn. Den Wind in den Kopf zu lassen. Stottern. Nichts sagen.« (Draesner 2015, 101) 
wie in den sieben als 100-Wörter-Bild überschriebenen Prosaabschnitten, die zum Prosagedicht hinneigen.

Der Prosaband steht in Kontinuität zu einer wiederholten Beschäftigung mit Hiddensee in Draesners Lyrik, etwa in dem Zyklus mittwinter von 2005 sowie in den beiden Hiddensee gewidmeten Gedichten hiddensee, südstrand, die kämpfenden vögel, veröffentlicht $2001 \mathrm{im}$ Gedichtband für die nacht geheuerte zellen (Draesner 2001, 64), und hiddensee, südstrand, die winkende bucht (hiddensee 2), erschienen im 2005 veröffentlichten Gedichtband kugelblitz. (Draesner 2005, $11 \mathrm{f}$.) ${ }^{17}$ In Kontinuität zu dem wiederkehrenden, autobiographisch motivierten Hiddensee-Thema stehen auch Draesners Äußerungen über die Bedeutung der Insel in ihrer Bamberger Poetikvorlesung Zielen. Zum Verhältnis von Wirklichkeit und Text (Draesner 2007, 89f.). Schon die Wiederkehr des Themas in unterschiedlichen Genres, von denen die Prosabearbeitung die (vorläufig) letzte Version darstellt, legt die sprachliche Verwandtschaft nahe, die Draesners Prosa zur Lyrik und zum Essay unterhält.

Draesners Texte - die lyrischen und die epischen ebenso wie die essayistischen - zeichnen sich, so Anna Ertel $(2014,19)$ in ihrem Beitrag zu Draesners Poetik, durch »Komplexität und Mehrdeutigkeit« sowie durch »eine ausgeprägte assoziative Bildlichkeit« aus. Ferner verweist Ertel auf »einen hohen Grad an metaphorischer Verdichtung«, das Arbeiten mit Mehrsprachigkeit, das Spiel mit Konnotationen und Assoziationen sowie auf ein Interesse am Unterminieren scheinbar binärer Kategorien und am Eigenwert poetischer Erkenntnis (ebd., 20-23). Dies mündet in »komplexe, hochgradig verdichtete und kunstvoll arrangierte Sprachgebilde«, so Ertel weiter, »die auf engstem Raum eine Vielzahl von Bild- und Bedeutungsbereichen versammeln und scheinbar mühelos Verbindungen zwischen heterogenen Diskursen (etwa Mythos und Naturwissenschaft) stiften « (ebd., 21). Draesners Prosa, insbesondere wie sie sich in Mein Hiddensee entfaltet, so die Argumentation, die ich an Ertels Analysen anschließen möchte, integriert die drei oben aufgezeigten Ebenen der Prosa als einer Gleichzeitigkeit von referentiellem, transzendentalem und sprachspielerisch-kombinatorischem Zeichengebrauch.

$\mathrm{Zu}$ diesen Verfahren zählen auch intertextuelle Verweise ${ }^{18}$ und kombinatorische Arrangements, die die Wörter sowohl auf einer semantischen als auch auf einer lautlichen Ebene miteinander verknüpfen. Intertextuelle Verweise unterhält etwa ein mit südstrand, winkende bucht überschriebenes Kapitel des Pro-

17 Zur Interpretation dieser Gedichte: Friedrich 2020 und Leeder 2008b, 139-144.

18 Mit Verfahren der Intertextualität hat sich Draesner (1993) bereits in ihrer Dissertation beschäftigt. 
sabandes zum Titel des zweiten Hiddensee-Gedichts hiddensee, südstrand, die winkende bucht. Doch die sich hier abzeichnenden Kontinuitäten der Sprachverwendung reichen weiter. Es sind Schreibweisen, die sich der Kombinatorik bedienen und Draesners Prosasammlung Mein Hiddensee ebenso bestimmen wie ihre Lyrik: Dazu zählt die für Draesners Lyrik vielfach konstatierte Lautmalerei, erzeugt durch Häufung von Alliterationen, Assonanzen und Anaphern, der Klimek (2020, 72) einen »litaneihaften«, eindringlichen Ton bescheinigt; dazu zählt auch das von Friedrich als fehlende Beschreibung wahrgenommene und von Klimek als »Aufzählung einzelner `Geräusch[e]< und sichtbarer Dinge« erfasste Verfahren, das »punktuell einen distanzierten Beobachter« (ebd., 72) erkennen lässt und die sinnliche Seite der Gegenstände mit Hilfe der sinnlichen Qualitäten der Sprache abzubilden versucht. Dieses Verfahren möchte ich exemplarisch anhand des letzten Kapitels von Mein Hiddensee genauer analysieren als eine Schreibweise der Prosa, in der sich die drei Ebenen des kombinatorisch-ähnlichkeitsbasierten, des transzendental-selbstreflexiven und des referentiell-inhaltlichen Zeichenbezugs überlagern.

Im letzten Kapitel gestaltet Draesner eine physische und eine reflexive Wanderung über die Insel und durch die Erinnerungsräume der Protagonistin. Erinnerungen an frühere Besuche des gleichen Ortes überlagern sich mit den sinnlichen Wahrnehmungen des aktuellen Erlebens. Das Kapitel arrangiert, wie der gesamte Text, wiederholt aufgegriffene und variierte Wörter und Phrasen zu immer neuen Wort-, Satz- und Textkonstellationen, in denen die Bedeutungen der Begriffe beständig aktualisiert werden und sich die dergestalt generierten Bedeutungsschichten basaltartig akkumulieren. Dabei entlockt Draesner der Sprache ihre poetischen Qualitäten, indem sie kombinatorische Verfahren in den Prosatext einträgt, die die Semantik der Wörter durch die klanglichen Eigenschaften überlagern. Dies geschieht etwa in der wiederholten und extensiven Einschreibung von Alliterationen in ihre Satzgefüge, die mal die Phänomenologie der sinnlich erfahrenen Landschaft in den Klang der Sprache zu übersetzen versucht, mal sprachspielerisch wie im Zungenbrecher Situationskomik in Sprachkomik überträgt: »Auf den Hügeln herrschen Westwinde, das Ufer säumten lila leuchtende Hagebutten. Hollerteller, Beeren, letztes Licht« (Draesner 2015, 191, Hervorhebungen Y.A.) oder in ludistischer Absicht: »Hindernisse wie herumliegende Handtücher werden von sandignassen Hundepfoten hübsch gemustert, Hindernisse wie auf herumliegenden Handtüchern herumliegende Menschen elegant, wenngleich tropfend übersprungen.« (ebd., 99f.) ${ }^{19}$ Die momenthafte sinnliche Erscheinung der beschriebenen

19 Im Folgenden werden Zitate aus diesem Text mit der Sigle MHS und der Angabe der Seitenzahl direkt im Lauftext nachgewiesen. 
Naturszenerie wiederholt sich im Ausstellen der sinnlichen Qualitäten des Sprachmaterials. Der Text verknüpft seine lexikalischen Elemente in immer neuen Konstellationen miteinander und streut Rekurrenzen teils über weite Textpassagen hinweg, teils nimmt er sie in rascher Abfolge wieder auf. So etwa »Harter Ort « (MHS, 61), das als Kapitelüberschrift auf einen gleichnamigen Küstenabschnitt der Insel verweist, der im ersten Satz des letzten Kapitels wieder Erwähnung findet: das »Meer« am »Harten Ort« (MHS, 191) und wenige Absätze weiter als Frage wiederaufgenommen auf die Semantik hinter dem Namen transparent gemacht wird: »Harter Ort? Das Meer.« (MHS, 191) Dieses Verfahren verdichtet sich im letzten Kapitel in einer Frage, die es in beständig variierender Wiederholung leitmotivisch durchzieht: »Was glücklich macht?« (MHS, 191, 192); »Was glücklich macht:« (MHS, 192); „Was glücklich macht.« (ebd.); »die glücklich macht« (MHS, 193); »Glücklich [...] Macht - was?« (ebd.); »Macht glücklich, wen?« (ebd.); »Glücklich:« (MHS, 194); »Glücklich [...]« (MHS, 195); »Glücklich sein:« (MHS, 197); »doch glücklich gewesen« (MHS, 198) und schließlich in der Perspektivverschiebung von der Insel als Ort oder Gegenstand der Glückssuche hin zur Insel als Agentin des Fragens mündet: »Die Insel selbst (schöne Einbildung) scheint ihr die Frage nach dem Glück zu stellen, zitternd, vielfältig rasch.« (MHS, 198). In diesem einkreisenden Suchen werden Antworten ausprobiert, die auf die beobachteten Phänomene der Inselflora bezogen werden können, die aber auch eine poetologische Lesart nahelegen, wonach die beschriebenen Wahrnehmungsbilder sich in den Zeichenrelationen spiegeln. ${ }^{20}$ Auf den Satz »[S]tändig tauschen Schatten und Helligkeit den Platz«, folgt die Frage: »Ist es Wiederholung, die glücklich macht? «(MHS, 192). Nicht nur im beschriebenen Wahrnehmungsphänomen des Licht- und Schattenwechsels ist die Wiederholung angelegt - der Satz selbst wiederholt die Aussage eines vorausgehenden Satzes: »Dämmerung und Licht werden in rascher Folge über den Weg geweht«. (MHS, 191) Und auch der Ellipse, die auf die Frage folgt, ist die Wiederholungsfigur in den Parallelismus und die Anaphorik ihrer Syntax eingeschrieben: »Rauschen des Meeres, Rauschen des Laubes.« (MHS, 192)

Der nächste Absatz lässt der Beschreibung verschlungen wuchernder Pflanzen: »Nessel- und Brombeerverhau, Dickicht durcheinanderwuchernder Pflanzen, eine zweite Kiefer, umrankt von einem efeuartigen, saugenden Wesen, streckt sich weit nach links Richtung Meer« die Frage folgen: »Was glücklich macht: Wachsen

\footnotetext{
$20 \mathrm{Zu}$ diesem Verfahren auch: Leeder 2008a, bes. 39, wo sie unter Bezugnahme auf Aussagen Draesners auf die doppelte - naturwissenschaftliche wie poetologische - Bedeutung des Begriffs „Kugelblitz« eingeht, oder van Hoorn (2018, 269), die bezogen auf den Gedichtband subsong vermerkt, dass sich in seinem Motto die »Parallelisierung von naturkundlicher Observation und Dichtungsproduktion« als »poetologisches Programm« des Bandes ankündigt, die sie als »Ornithopoetik« bezeichnet.
} 
in alle Richtungen?« (MHS, 192) Auch dieser Satz legt eine poetologische Lesart nahe, die an das Textmodell des Rhizoms erinnert und an die darin angelegte Lösung der Weltwahrnehmung von herrschenden Ordnungsmustern. ${ }^{21}$ Es folgen Antworten, die sich in ähnlicher Weise sowohl auf die Inhalte wie auf die poetischen Verfahren des Textes beziehen lassen: »Ist es die Erinnerung, die glücklich macht, oder das Bildermachen, ist es das Wiedererkennen oder der Versuch, einfach nur hier zu sein« (MHS, 193). Erinnern, Bildermachen, Wiedererkennen und schließlich der Versuch, präsentisches Erleben darzustellen, sind Schreibverfahren, die den Text durchziehen.

Im weiteren Verlauf des Kapitels wird das Verhältnis von Eigenschaften beschriebener Natur zu den sie bezeichnenden Worten zum Thema. Dergestalt überführt der Text die epistemisch-referentielle Ebene auf eine metareflexivselbstreferentielle, die über das Verhältnis von Zeichen und Bezeichnetem reflektiert:

Dunkelrote Beeren an einem Busch mit rundlichen, bemehlten Blättern. Die Beeren leuchten durchsichtig.

Durchsichtig: das Deutsche und die Anschaulichkeit seiner Wörter. Schubkarre. Butterblume. Wetterhahn. Das Deutsche und seine von Bildlichkeit geleiteten Wortzusammensetzungen. Dazu die oft einsilbigen Grundvokabeln. Sie erschließen sich nicht, sie muss man lernen: Kind Mann Frau Kuh Gras Mensch Tat Werk Tier Haus Tür Bett Stall Topf Herd Hand Kopf Bein Arm Fuß Mund Heu Meer See Baum Strauch Busch Ei Loch. Fügt man sie zusammen, wird sofort deutlich (deutlich), was sie bedeuten (was sie sein könnten?): Leuchtturm. Grastafel. Enddorn.

(MHS, 196)

Wie in der frühneuzeitlichen Kombinatorik stellt Draesner hier Wortlisten zusammen, die sich einerseits zu Wortfeldern gruppieren, andererseits über die Wortfeldgrenzen hinweg a-semantisch klangliche Beziehungen untereinander unterhalten, etwa in den wiederkehrenden Alliterationen (Tat/Tier/Tür/Topf, Herd/Hand), den Binnenreimen (Tier/Tür, Topf/Kopf) und den Assonanzen (Fuß/Mund, Meer/See, Baum/Strauch). Die hier ausgebreitete Sprachreflexion lässt an die ıSpracharbeit (Hundt 2000, Titel) der Frühen Neuzeit denken, die ihren Ausgang beim Stammwort nimmt und in ludistischen Formen Wortlisten durch Kompositabildungen, Flexionen und Ableitungen generiert (ebd., 272-279). Die »Kombinierlust« (ebd., 277) vermischt sich bei Draesner mit einer Aufmerksamkeit für das bildliche Potential der Komposita, die sie auf ihren Referenzbereich hin durchsichtig macht. Diese Aufmerksamkeit für das bildliche Potential deutscher Komposita durchzieht den Text. In einem der ersten Kapitel reflektiert Draesner die Bezeich-

21 Die Wahrnehmungsweise erinnert bisweilen an Paul Cézannes neuen Blick auf die Landschaft, auf den sich Deleuze wiederholt bezieht (Lehmann 1984, 544). 
nung, nach der sie umschreibend sucht: »Es gibt einen Namen für diese windgeformten Gestalten; wie Fühler strecken sie ihre Äste in die Luft.« (MHS, 10) Da, wo das Wort - fünfzehn Seiten und zwei Kapitel weiter - wieder ins Bewusstsein und in den Text tritt, reduzieren sich die Bildlichkeit und Klanglichkeit der umschreibenden Sprache, um sich ganz im Kompositum des Begriffs zu verdichten: „Windflüchter heißen die schiefen Bäume, jetzt fällt es ihr ein.« (MHS, 25) Semiotische Kombinatorik, selbstreflexiver Sprachbezug und Repräsentationsmodell treten hier zusammen und verdichten die Ebenen des Textes.

Enzyklopädisches Denken und Topoi vom Buch der Natur werden aufgerufen, nur um unterminiert zu werden. Natur und Buch stehen in keiner analogen Ähnlichkeitsrelation zueinander, stattdessen werden im Sprechen über die Natur zwei unabhängige Zeichensysteme miteinander korreliert, die ihren eigenen Gesetzlichkeiten folgen:

Sie schlägt die Namen der Pflanzen nach. `Die Natur` ist kein Buch, das Buch wird über ihre Erscheinungen gelegt. Im Augenblick: Vokabular. Von der Grammatik, den Regeln der Verknüpfung (genannt Biotop, Soziotop, Biosphäre, Reservat, Schutz, $\mathrm{CO}_{2}$-Fußabdruck) versteht sie nicht viel.

(MHS, 28)

Das Wörterbuch dient immer wieder dem Ordnen und Artikulieren der Seheindrücke und stellt dabei die akustische und kombinatorische Eigenlogik der Sprache aus: »Ancylus fluviatilis, Littorina littorea, Limnea ovata, Mya arenaria. Flussmützenschnecke. Strandschnecke. Brackwasserschnecke, Sandklaffmuschel.« (MHS, 69) Das Lexikon wird in seiner Funktion der Kartierung der Umwelt ebenso mitreflektiert wie die kombinatorische Verwandtschaft fachterminologischer Komposita oder die im Synonym geborgene Vervielfältigung der Signifikanten: «Noch lernt er Vokabeln: Steilküste, Flachküste, Nehrung, Windwatt. Eiche, Buche, Kiefer, Schwein. Die Namen der Büsche enden alle gleich: Weißdorn, Rotdorn, Kreuzdorn, Sanddorn.« (MHS, 103) Oder: »Namen der Königskerze: Wollkraut, Kunkel, Wullena, Blitz.« (MHS, 95)

Die strukturelle Ironie entfaltet sich zwischen den fachterminologisch präzisen Bestimmungen und Benennungen der Inselflora und deren Arrangement zu kombinatorisch organisierten Wortfolgen, in denen die Bildlichkeit der Wörter, die Zusammensetzung aus sich wiederholenden Sprachbausteinen und klanglich a-semantische Qualitäten der Sprache hervortreten. Dergestalt wird eine im Fachterminus archivierte Wissensformation überschrieben und gebrochen durch eine $\mathrm{zu}$ ihr scheinbar in Ko-Opposition stehende poetische Wissensordnung der sinnlich wahrnehmbaren Erscheinungen der Natur, die in die sinnlich-onomatopoetischen Qualitäten der Sprache hineingespiegelt wird. Die Inselflora wird dergestalt auf zweifache Weise kartiert: in den Epistemen der Repräsentation, der die Fachterminologie folgt, und in einer ähnlichkeitsbasierten Episteme, die auf die kom- 
binatorischen Spracharrangements als Abbild sinnlich erfahrbarer Wirklichkeit zielt. Es ist schließlich die dritte, transzendentale Episteme, die zwischen diesen beiden Epistemen vermittelt und dabei das Verhältnis der Zeichen zu den Gegenständen reflektiert.

So gilt für Draesners Prosa gerade nicht, was Sonja Klimek über ihre Lyrik in Abgrenzung zur Prosa schreibt: „Der >Mehrwert` des Gedichts gegenüber einem autobiographischen Erlebnisbericht in Prosa beispielsweise ist bewirkt durch die generisch besondere, sinnlich wirkende Sprachverwendung als zum rein propositionalen Inhalt [...] hinzutretende, nicht-propositionale, graphische und akustische Erfahrungsqualität des Textes.«(Klimek 2020, 81)

Mit Mein Hiddensee schreibt Ulrike Draesner einen Prosatext, der im weitesten Sinne als sautobiographischer Erlebnisbericht ‘ gelten könnte und der sich doch gerade nicht auf propositionale Aussagegehalte reduzieren lässt, sondern vielmehr basierend auf einer kombinatorischen Schreibweise unterschiedlichste propositionale Inhalte (botanisches Fachwissen, historisches Wissen, persönliche Erinnerungen, überlieferte Erinnerungen) und die nicht-proportionalen Eigenschaften der Zeichen rhizomatisch verschaltet, das doppelte Potential der Sprache dabei beständig mitreflektiert und auf diese Weise einen Prosatext gestaltet, der die semiotischen Interdependenzen auf der Ebene der Inhalte, der Materialität der Zeichen und der Selbstreferenz zu einer nachgerade lyrischen Dichte ${ }^{22}$ steigert.

\section{Literaturverzeichnis}

Arndt, Astrid, Christoph Deupmann und Lars Korten (Hg.): Logik der Prosa. Zur Poetizität ungebundener Rede. Göttingen 2012.

Blumenberg, Hans: Theorie der Unbegrifflichkeit. Aus dem Nachlass hrsg. v. Anselm Haverkamp. Frankfurt a. M. 2007.

Breitbach, Olaf: Die Typik des Wissens und die Ordnung der Dinge. Zur Systematik des Goetheschen Sammelns. In: Räume der Kunst. Blicke auf Goethes Sammlungen. Hrsg. v. Markus Bertsch und Johannes Grave. Göttingen 2005, 322-341.

Dan, Joseph: The Kabbalah of Johannes Reuchlin and its historical significance. In: The Christian Kabbalah. Jewish mystical books and their Christian interpreters. Hrsg. v. Joseph Dan. Cambridge, MA 1997, 55-95.

Deleuze, Gilles und Félix Guattari: Rhizom. Aus dem Französischen von Dagmar Berger, Clemens-Carl Haerle, Helma Konyen, Alexander Krämer, Michael Nowak und Kade Schacht. Berlin 1977.

22 Hier setzen auch die Überlegungen Walter Benjamins zur Prosa als »Reflexionsmedium« und "Schöpfungsgrund « der Poesie an, die Ralf Simon zum Ausgangspunkt seiner Studie Die Idee der Prosa wählt (Simon 2013, 9-12). 
Draesner, Ulrike: Wege durch erzählte Welten. Intertextuelle Verweise als Mittel der

Bedeutungskonstitution in Wolframs >Parzivalı. Frankfurt a. M. 1993.

Draesner, Ulrike: für die nacht geheuerte zellen. Gedichte. München 2001.

Draesner, Ulrike: Kugelblitz. Gedichte. München 2005.

Draesner, Ulrike: Zielen. Zum Verhältnis von Wirklichkeit und Text. In: dies.: Zauber im Zoo.

Vier Reden von Herkunft und Literatur. Göttingen 2007, 83-107.

Draesner, Ulrike: Mein Hiddensee. Hamburg 2015.

Draesner, Ulrike: Vom zärtlichen Ernst der Welt. Nature Writing. In: dies.: Grammatik der

Gespenster. Frankfurter Poetikvorlesungen. Stuttgart 2018, 155-189.

Ernst, Ulrich: Permutation als Prinzip in der Lyrik. In: Poetica 24.3 (1992), 225-269.

Ertel, Anna: Zur Poetik Ulrike Draesners. In: Ulrike Draesner. Text und Kritik 201 (2014), 19-26.

Foucault, Michel: Die Ordnung der Dinge. Eine Archäologie der Humanwissenschaften.

Aus dem Französischen v. Ulrich Köppen. Frankfurt a. M. 1974.

Frank, Manfred: Einführung in die frühromantische Ästhetik. Vorlesungen. Frankfurt a. M. 1989.

Friedrich, Hans-Edwin: Ulrike Draesner. hiddensee, südstrand, die winkende bucht (hiddensee

2). In: Gedichte von Ulrike Draesner. Interpretationen. Hrsg. v. Christoph Jürgensen, Erik

Schilling und Rüdiger Zymner. Leiden u. a. 2020, 99-109.

Hoorn, Tanja van: Zwischen Anmerkungslust und Reflexionszwang. Poetologische Paratexte in aktuellen Lyrikbänden. In: Zeitschrift für Germanistik 28.2 (2018), 261-274.

Hundt, Markus: „Spracharbeit« im 17. Jahrhundert. Studien zu Georg Philipp Harsdörffer, Justus Georg Schottelius und Christian Gueintz. Berlin 2000.

Klimek, Sonja: »fehlt das kind | - im bauch«. Ulrike Draesners Zyklus bläuliche sphinx (metall). In: Gedichte von Ulrike Draesner. Interpretationen. Hrsg. v. Christoph Jürgensen, Erik Schilling und Rüdiger Zymner. Leiden u. a. 2020, 67-81.

Koschorke, Albrecht: Wahrheit und Erfindung. Frankfurt a. M. 2012.

Lansius, Thomas: Orationes seu consultatio de principatu inter provincias Europae. Praefatio ad lectorem. Tübingen 1620 .

Leeder, Karen: Eine Grammatik der Liebe. Ulrike Draesners Lyrik. In: Familien, Geschlechter, Macht. Beziehungen im Werk Ulrike Dreasners. Hrsg. v. Stephanie Catani und Friedhelm Marx. Göttingen 2008a, 37-59.

Leeder, Karen: Heimat in der neuen deutschen Lyrik. In: Gedächtnis und Identität. Die deutsche Literatur nach der Vereinigung. Hrsg. v. Fabrizio Cambi. Würzburg 2008b, 135-153.

Lehmann, Hans-Thies: Rhizom und Maschine. Zu den Schriften von Gilles Deleuze und Félix Guattari. In: Merkur 38.427 (1984), 542-550.

Neubauer, John: Symbolismus und symbolische Logik. Die Idee der Ars Combinatoria in der Entwicklung der modernen Dichtung. München 1978.

Roßbach, Regina: Von allem zu viel. »Mein Hiddensee« ist Ulrike Draesners Versuch, ihre Erinnerungen von der Natur überwuchern zu lassen. In: literaturkritik.de (3.9.2015). 5. August 2020: https://literaturkritik.de/id/21090 [letzte Änderung: 21.11.2016].

Schlegel, Friedrich: Kritische Friedrich-Schlegel-Ausgabe. Hrsg. v. Ernst Behler et al. Paderborn u. a. $1958 \mathrm{ff}$. [zitiert als KFSA].

Simon, Ralf: Die Idee der Prosa. Zur Ästhetikgeschichte von Baumgarten bis Hegel mit einem Schwerpunkt bei Jean Paul. München 2013.

Simon, Ralf: Durcheinanderprosa (Jean Paul, Wilhelm Raabe, Arno Schmidt). In: Jahrbuch der Jean-Paul-Gesellschaft 48/49 (2014), 19-37.

Zovko, Jure: Ironie, Witz. In: Friedrich Schlegel-Handbuch. Leben - Werk - Wirkung. Hrsg. v. Johannes Endres. Stuttgart 2017, 309-312. 